\title{
Metallicity and kinematics of a large sample of LMC and SMC clusters
}

\author{
A. J. Grocholski ${ }^{1}$, M. C. Parisi ${ }^{2}$, D. Geisler ${ }^{3}$, A. Sarajedini ${ }^{4}$, \\ A. A. Cole ${ }^{5}$ J. J. Clariá ${ }^{2}$ and V. V. Smith \\ ${ }^{1}$ Space Telescope Science Institute, USA \\ ${ }^{2}$ Observatorio Astronómico, Universidad Nacional de Córdoba, Argentina \\ ${ }^{3}$ Departamento de Astronomía, Universidad de Concepción, Chile \\ ${ }^{4}$ Department of Astronomy, University of Florida, USA \\ ${ }^{5}$ University of Tasmania, Australia \\ ${ }^{6}$ Gemini Project, National Optical Astronomy Observatory, USA
}

\begin{abstract}
We have carried out a large-scale investigation of the metallicity and kinematics for a number of LMC and SMC star clusters using Ca II triplet spectra obtained at the VLT. Our sample includes $28 \mathrm{LMC}$ and $16 \mathrm{SMC}$ clusters, covering a wide range of ages and spatial extent of the host galaxy. We determine mean cluster velocities to about $2 \mathrm{~km} \mathrm{~s}^{-1}$ and metallicities to 0.05 dex (random error), from about 7 members per cluster. Herein we present the main results for this study for the cluster metallicity distributions, metallicity gradients, age-metallicity relations and kinematics.
\end{abstract}

Keywords. stars: kinematics, Magellanic Clouds, galaxies: star clusters

\section{Introduction}

Star clusters in the LMC and SMC are of fundamental importance for a variety of reasons. On a cosmological scale, star clusters in the Magellanic Clouds are necessary for the understanding of stellar populations in distant galaxies since they occupy regions of the age-metallicity plane that are devoid of Milky Way clusters. Thus, accurate knowledge of their properties is needed to properly employ LMC and SMC clusters as tests of stellar evolution models as well as empirical templates of simple stellar populations. For the LMC and SMC specifically, their populous star clusters, which preserve a record of their host galaxy's chemical abundances at the time of their formation, provide the most straightforward means of determining the star formation and chemical enrichment histories of these two galaxies.

Despite their utility, the LMC and SMC cluster systems have surprisingly little up-todate information available. The only previous large scale study of cluster abundances and velocities in the LMC was performed by Olszewski et al. (1991); they observed the Ca II triplet (CaT) lines in RGB stars in $\sim 80$ LMC clusters. However, the accuracy of their results was limited by the technology ( $4 \mathrm{~m}$ class telescopes with single-slit spectrographs) available at the time. For the SMC, the situation is worse, with only two clusters having abundances based on high-resolution spectra (NGC 330 - Hill 1999; NGC 121 - Johnson et al. 2004) and an additional six with CaT based velocities and metallicities (Da Costa \& Hatzidimitriou 1998, DH98). To improve upon our knowledge of the abundances and kinematics of star clusters in the Magellanic Clouds, we have taken advantage of the multiplexing capability of modern spectrographs and obtained CaT spectra for hundreds of individual RGB stars spread across 28 LMC and 16 SMC clusters. Herein we provide a 
brief description of our work, while the full details can be found in Grocholski et al. (2006, hereafter G06) and Parisi et al. (2008, hereafter P08) for the LMC and SMC, respectively.

\section{Data}

Spectroscopic observations of our LMC clusters were carried out in December 2004, and our SMC targets were observed in November 2005. In both cases we used the FORS2 spectrograph in mask exchange unit mode on the Antu (VLT-UT1) $8.2 \mathrm{~m}$ telescope at ESO's Paranal Observatory. Our spectra were centered on the CaT lines $(\sim 8500-8700$ $\AA$ ), with a resolution of $2-3 \AA$. Data processing was carried out within the IRAF environment. Using standard IRAF tasks, we flat-fielded the images, fixed bad pixels, corrected the images for distortions, and extracted and normalized the stellar spectra. The resulting $\mathrm{S} / \mathrm{Ns}$ were typically $25-50 \mathrm{pixel}^{-1}$. Radial velocities for all stars were determined via cross-correlation with 30 template stars using the IRAF task fxcor (Tonry \& Davis 1979). We find a good agreement amongst the template-derived velocities, with a standard deviation of typically $\sim 6 \mathrm{~km} \mathrm{~s}^{-1}$ for each star.

We measure the equivalent width of each CaT line as follows. Since the near-infrared portion of a star's spectrum can be contaminated by weak metal lines (and possibly weak molecular bands), measuring the true equivalent width of the CaT lines at moderate resolution is virtually impossible. Instead, we follow the method of Armandroff \& Zinn (1998) by defining continuum bandpasses on each side of each CaT line and then measuring the "pseudocontinuum" as a linear fit to the mean value in each pair of continuum windows. The "pseudo-equivalent width" is then determined by fitting the sum of a Gaussian and a Lorentzian to each CaT line with respect to the pseudocontinuum. Note that, for some low S/N stars in SMC clusters, we have fit only a Gaussian (see P08 for more details). We adopt the same definition for the summed equivalent width, $\Sigma W$, as Cole et al. (2004), namely,

$$
\Sigma W \equiv E W_{8498}+E W_{8542}+E W_{8662} .
$$

While both theoretical (Jørgensen, Carlsson \& Johnson 1992) and empirical (Cenarro et al. 2002) studies have shown that effective temperature, surface gravity, and metallicity all play significant roles in determining CaT line strengths, it is well established that there is a linear relationship between a star's absolute magnitude and $\Sigma W$ for red giants of a given metallicity. Similar to previous authors, we remove the effects of luminosity and temperature on $\Sigma W$ by defining a reduced equivalent width, $W^{\prime}$, as

$$
W^{\prime} \equiv \Sigma W+\beta\left(V-V_{H B}\right)
$$

where the introduction of the brightness of the cluster's horizontal branch, $V_{\mathrm{HB}}$, removes any dependence on cluster distance or reddening as well. For intermediate age clusters without fully formed HBs, we adopt the median value of their core helium burning red clump stars in place of $V_{\mathrm{HB}}$. Since our target clusters span a range of ages and metallicities similar to the calibration clusters used by Cole et al. (2004), we adopt their value of $\beta=0.73$. Finally, as shown by Rutledge et al. (1997) for Milky Way globular clusters, there is a linear relationship between a cluster's reduced equivalent width and its abundance on the Carretta \& Gratton (1997) metallicity scale. Cole et al. (2004) extended this relation to a larger range of abundances and ages, and so we adopt their relationship,

$$
[\mathrm{Fe} / \mathrm{H}]=(-2.966 \pm 0.032)+(0.362 \pm 0.014) W^{\prime} .
$$

Cluster members are isolated from field stars using a combination of three criteria: distance from the cluster center, radial velocity, and metallicity. For the LMC, we identify 
an average of eight members per cluster and determine mean cluster velocities to typically $1.6 \mathrm{~km} \mathrm{~s}^{-1}$ and mean metallicities to $0.04 \mathrm{dex}$ (random error). Similarly, for the SMC we find an average of 6.4 cluster members, and calculate mean cluster velocities and metallicities to $2.7 \mathrm{~km} \mathrm{~s}^{-1}$ and 0.05 dex, respectively. For eight of our LMC clusters and all of our SMC clusters, we report the first spectroscopically derived metallicity and radial velocity values based on individual stars within these clusters.

We note that, while for the LMC our results are based solely on our CaT data sample, for the SMC we have enlarged our cluster sample size by including the abundances and velocities derived from the CaT by DH98 for 6 clusters and photometric abundances for 5 clusters obtained by Piatti et al. (2001, 2007; see P08 for more details).

\section{Results}

Kinematics. The LMC is known to have primarily a disk-like structure, with little evidence of a halo component. This has been show through studies of the LMC's H I gas (Kim et al. 1998), field stars (e.g., van der Marel \& Cioni 2001) and populous clusters (Grocholski et al. 2007). Our cluster kinematics (see Fig. 3) are in excellent agreement with the work of Schommer et al. (1992) and confirm that all of the clusters so far studied, including our eight "new" targets, have disk-like kinematics with no obvious signature of the existence of a pressure-supported halo.

In contrast, the SMC appears to have a much more complex structure. Distances derived from photometry of star clusters show that the SMC has a large line-of-sight depth that may vary across the face of the galaxy (Crowl et al. 2001). Kinematics in the SMC, however, give mixed results. Stanimirović et al. (2004) found differential rotation in the SMC's H I gas and suggest that the inner $3 \mathrm{kpc}$ may correspond to a disk-like structure left over from when the SMC was rotationally supported. On the other hand, stellar kinematics indicate that the SMC is likely a pressure supported system (e.g., Harris \& Zartisky 2006). While our clusters show a hint of possible rotation (see Fig. 15 in P08), the velocity amplitude $\left(\sim 10 \mathrm{~km} \mathrm{~s}^{-1}\right)$ is considerably smaller than the dispersion $(\sim 21$ $\mathrm{km} \mathrm{s}^{-1}$ ), and thus our results agree with previous work in that the stellar component of the SMC is in a pressure-supported state.

Metallicity. The metallicity distribution, whether derived from individual stars or clusters, is an important diagnostic of the global chemical enrichment of a galaxy, and provides a straightforward way of comparing stellar populations in different galaxies. In Fig. 2 we plot the metallicity distribution of both our LMC (top panel) and SMC (bottom panel) cluster samples. With the LMC, we see a few of the well-known old metal-poor clusters, and a very tight metallicity distribution for the more metal-rich clusters, with a mean $[\mathrm{Fe} / \mathrm{H}]=-0.48$ and $\sigma=0.09$. On the other hand, the entire SMC cluster sample shows a very broad distribution of abundances, with mean $[\mathrm{Fe} / \mathrm{H}]=-1.00$ and $\sigma=$ 0.20 , and the hint of a possible bimodal distribution, although a larger sample is needed to explore this further. There are also two other key differences between the metallicity distribution in the LMC and SMC. First is the fact that the most metal-poor cluster in the SMC, NGC 121, is > 0.5 dex more metal-rich than the most metal-poor clusters in the LMC. And the second is that the most metal-rich clusters in the LMC are $\sim 0.3$ dex more metal-rich than the ones in the SMC.

Since we are dealing with clusters, for which ages are readily determined, we can further explore the metallicity distribution by creating age-metallicity relations (AMRs) for both the LMC and SMC. In the top panel of Fig. 3 we plot the AMR for the LMC, where we have assumed that the old metal-poor clusters are all coeval (13 Gyr), and the ages of the remaining clusters are obtained from main sequence turnoff fitting (Grocholski 


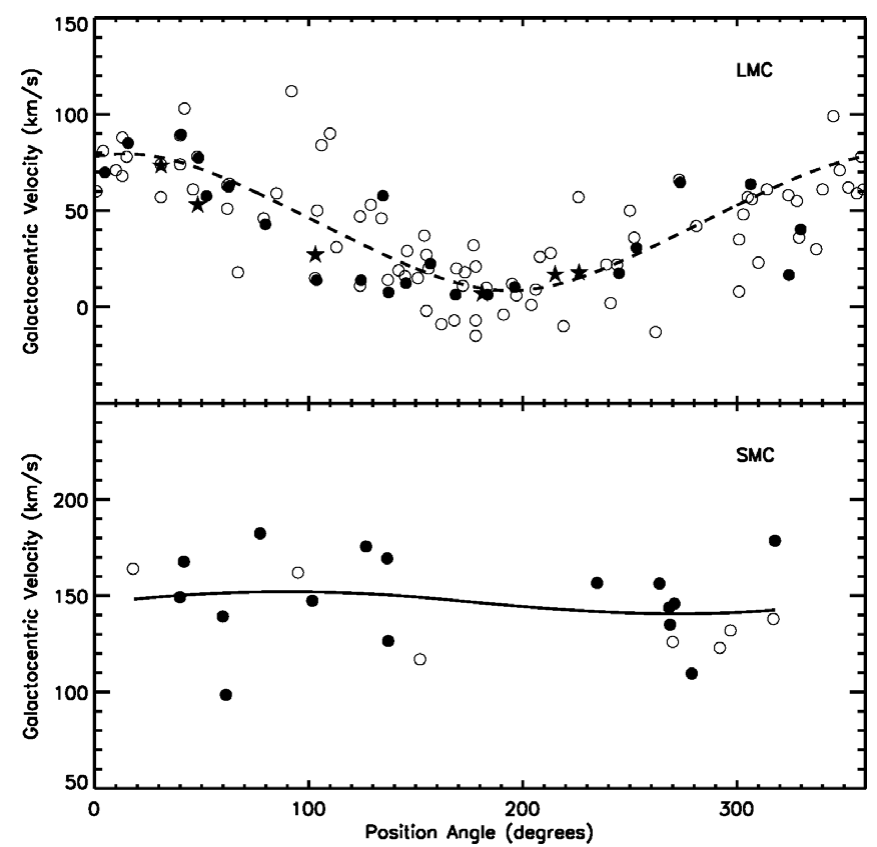

Figure 1. Radial velocity plotted as a function of position for the LMC and SMC. In the top panel, open circles are the data from Schommer et al. (1992), while the filed symbols are from G06. The dashed curve represents the best-fit rotation curve from Schommer et al. (1992). In the bottom panel, filled symbols are data from $\mathrm{P} 08$ while the open symbols are clusters from DH98. The best fit to the SMC data is shown as a solid curve.

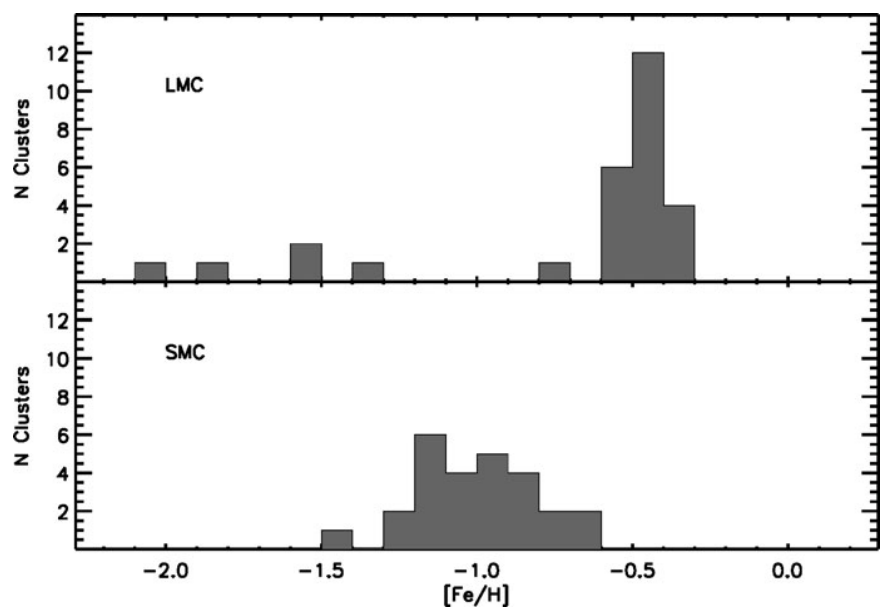

Figure 2. Metallicity distribution function of populous clusters in the LMC and SMC.

et al. these proceedings). The dashed line represents the smooth chemical enrichment model of Pagel \& Tautvaišienè (1998) and the solid line is their bursting model. While the well known age gap in the LMC makes it difficult to identify the best fit model for ages older than $\sim 4 \mathrm{Gyr}$, the intermediate age clusters suggest that the bursting model is the best representation of the LMC's chemical enrichment history. In the SMC's AMR (bottom panel of Fig. 3), we see a more complicated situation. The oldest cluster in the SMC, NGC 121, is only slightly younger (12 Gyr) than the oldest clusters in the 


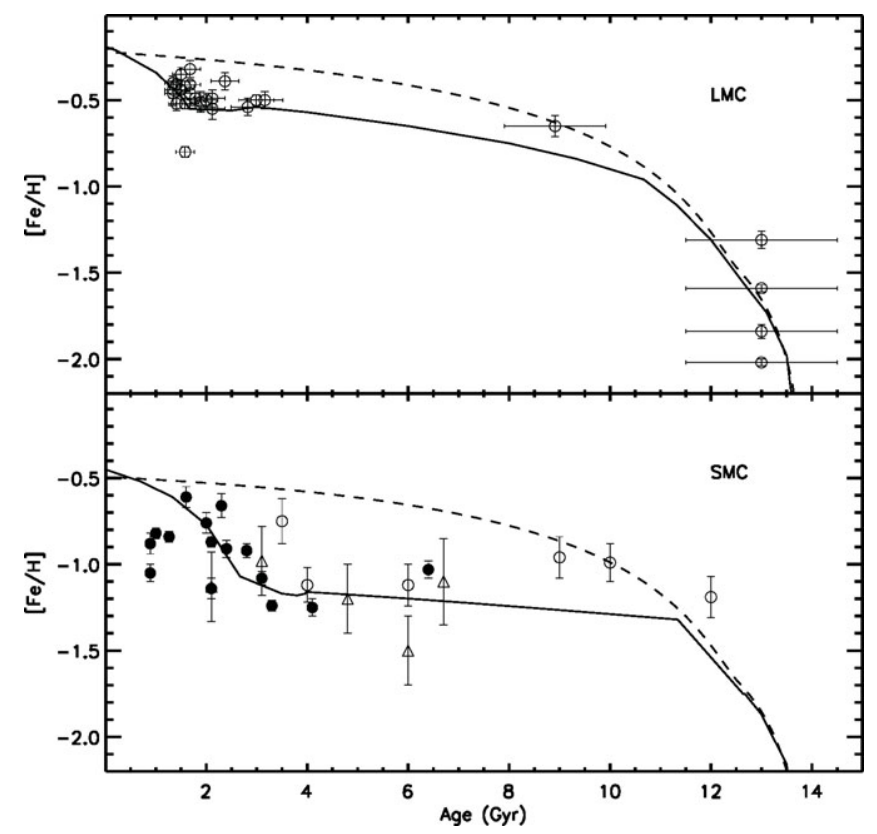

Figure 3. Age - metallicity relations for both the LMC and SMC. The dashed and solid lines represent the smooth and bursting chemical evolution models of Pagel \& Tautvaišienè (1998), respectively. For the SMC, we have supplemented our CaT data (filled symbols) with data from DH98 (open circles) and Piatti et al. (2001, 2007; open triangles).

LMC, yet is has an abundance that is roughly 1 dex more metal-rich than the metalpoor LMC clusters, which indicates that the SMC was "pre-enriched" prior to the start of cluster formation. From the formation of NGC 121 up until about 4 Gyr ago, SMC clusters showed very little enrichment, and while the clusters younger than $\sim 4$ Gyr are generally more consistent with a bursting chemical enrichment history (solid line), there is still considerable scatter amongst these clusters, with a mean that is only a few tenths of a dex higher than for the oldest clusters. Our LMC and SMC results reinforce the mysterious situation where the "LMC managed to make metals but no clusters during the age gap while the SMC managed to make clusters but no metals" (Da Costa 1991).

Zaritsky et al. (1994) studied H II region oxygen abundances in 39 galaxies and found that disk abundance gradients are ubiquitous in spiral galaxies. However, the presence of a classical bar (one that extends over a significant fraction of the disk length) tends to weaken the gradient. Both the LMC and SMC possess strong stellar bars and, as expected, neither galaxy's cluster system exhibits an abundance gradient (see Fig. 10 in G06 and Fig. 13 in P08).

\section{Summary}

We have used the multi-object spectrograph, FORS2, on the VLT to obtain moderateresolution near-infrared spectra of the Ca II triplet lines in individual red giant branch stars in a sample of LMC and SMC clusters. These data have allowed us to derive radial velocities and abundances for $\sim 7$ members per cluster with small random errors $\left(2 \mathrm{~km} \mathrm{~s}^{-1}\right.$ in velocity and 0.05 dex in metallicity). The main results of our study are as follows: 
1. The intermediate-age clusters in our LMC sample show a very tight distribution, with a mean $[\mathrm{Fe} / \mathrm{H}]=-0.48$ and $\sigma=0.09$. In contrast, the intermediate-age SMC clusters have a more metal-poor and much wider distribution, with $[\mathrm{Fe} / \mathrm{H}]=-0.88$ and $\sigma=0.17$.

2. For both the SMC and LMC, we find no evidence of a radial metallicity gradient. This is consistent with the work of Zaritsky et al. (1994) and suggests that the bar in each galaxy is responsible for the well-mixed cluster systems.

3. Age-metallicity relations for each galaxy show that the cluster formation histories are more consistent with a bursting chemical enrichment model than a smooth one.

4. We find that our radial velocities for the LMC clusters are in excellent agreement with the work of Schommer et al. (1992), and confirm that the LMC cluster system has disk-like kinematics, with no obvious signature of a halo population. The SMC, on the other hand, shows no obvious signature of rotation, and is thus likely a pressure supported system. The kinematics of both galaxies match their stellar distributions, which are known to be disk-like for the LMC, while the SMC has a large (and varying) line-ofsight depth.

\section{References}

Armandroff, T. E. \& Zinn, R. 1988, AJ, 96, 92

Cenarro, A. J., Gorgas, J., Cardiel, N., Vazdekis, A., \& Peletier, R. F. 2002, MNRAS, 329, 863 Carretta, E. \& Gratton, R. G. 1997, A\&BA, 121, 95

Cole, A. A., Smecker-Hane, T. A., Tolstoy, E., Bosler, T. L., \& Gallagher, J. S. 2004, MNRAS, 347,367

Crowl, H. H., Sarajedini, A., Piatti, A. E., Geisler, D., Bica, E., Clariá, J. J., \& Santos, J. F. C. Jr. 2001, $A J, 122,220$

Da Costa, G. 1991, in R. Haynes \& D. Milne (eds.), IAUS 148, The Magellanic Clouds, p. 143

Da Costa, G. S. \& Hatzidimitriou, D. 1998, AJ, 115, 1934 (DH98)

Grocholski, A. J., Cole, A. A., Sarajedini, A., Geisler, D., \& Smith, V. V. 2006, AJ, 132, 1630 (G06)

Grocholski, A. J., Sarajedini, A., Olsen, K. A. G., Tiede, G. P., \& Mancone, C. L. 2007, AJ, 134,680

Harris, J. \& Zaritsky, D. 2006, AJ, 131, 2514

Hill, V. 1999, A\&̊A, 345, 430

Johnson, J. A., Bolte, M., Hesser, J. E., Ivans, I. I., \& Stetson, P. B. 2004, in A. Mc William \& M. Rauch (eds.), Carnegie Observatories Astrophysics Series 4, p. 29

Jørgensen, U. G., Carlsson, M., \& Johnson, H. R. 1992, A\& A, 254, 258

Kim, S., Staveley-Smith, L., Dopita, M. A., Freeman, K. C., Sault, R. J., Kesteven, M. J., \& McConnell, D. 1998, ApJ, 503, 674

Olszewski, E. W., Schommer, R. A., Suntzeff, N. B., \& Harris, H. C. 1991, AJ, 101, 515

Pagel, B. E. J. \& Tautvaišienè, G. 1998, MNRAS, 299, 535

Parisi, M. C., Grocholski, A. J., Geisler, D., Sarajedini, A., \& Clariá, J. J. 2008, AJ, submitted [arXiv:0808.0018] (P08)

Piatti, A. E., Santos, J. F. C., Jr., Clariá, J. J., Bica, E., Sarajedini, A., \& Geisler, D. 2001, MNRAS, 325, 792

Piatti, A. E., Sarajedini, A., Geisler, D., Gallart, C., \& Wischnjewsky, M. 2007, MNRAS, 381, L84

Rutledge, G. A., Hesser, J. E., Stetson, P. B., Mateo, M., Simard, L., Bolte, M., Friel, E. D., \& Copin, Y. 1997, PASP, 109, 883

Schommer, R. A., Olszewski, E. W., Suntzeff, N. B., \& Harris, H. C. 1992, AJ, 103, 447

Stanimirović, S., Staveley-Smith, L., \& Jones, P. A. 2004, ApJ, 604, 176

Tonry, J., \& Davis, M. 1979, AJ, 84, 1511

van der Marel, R. P. \& Cioni, M.-R. L. 2001, AJ, 122, 1807

Zaritsky, D., Kennicutt, R. C., Jr. \& Huchra, J. P. 1994, ApJ, 420, 87 